\title{
Left ventricular function, aortic velocity, and late gadolinium enhancement assessed by real-time and single shot CMR is comparable to breath- held segmented imaging: a prospective study
}

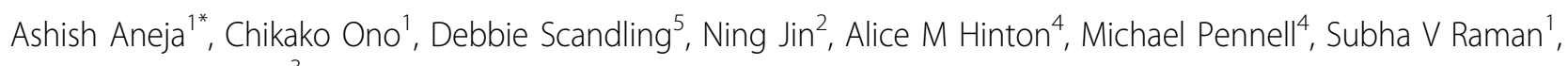
Orlando P Simonetti ${ }^{3}$

From 16th Annual SCMR Scientific Sessions

San Francisco, CA, USA. 31 January - 3 February 2013

\section{Background}

The typical CMR exam utilizes segmented k-space acquisitions that require repeated breath-holds, a regular cardiac rhythm, and long exam times. Widespread utilization of CMR has been hampered by prolonged exam times that limit cost-effectiveness, and limited reliability in patients with irregular rhythm and/or inability to breath-hold.

\section{Objectives}

The purpose of this study was to prospectively compare assessments of left ventricular (LV) ejection fraction (EF), aortic velocity, and late gadolinium enhancement by single-shot and real time (RT) techniques vs. traditional breath-hold segmented k-space (BH) acquisitions.

\section{Methods}

Thirteen patients referred to the CMR lab for evaluation of undiagnosed cardiomyopathy were prospectively enrolled. All patients were in regular cardiac rhythm and able to breath-hold. Standard segmented k-space images (including cine, velocity mapping (VM), and late-gadolinium enhancement (LGE)) were acquired during breathhold; real-time images (including cine, VM, and LGE) were acquired during free-breathing. Relevant imaging parameters are listed in Table 1. Breath-held and real-time studies for LGE were blinded and scored on a segmental basis for presence or absence of enhancement by two

Department of Internal Medicine, Department of Cardiovascular Medicine, The Ohio State University Medical Center, Columbus, OH, USA

Full list of author information is available at the end of the article reviewers. LVEF was calculated using Simpson's rule on short-axis $\mathrm{BH}$ cines, and biplane area-length methodology on long-axis RT cines. EF results were averaged across cardiac cycles when RT cine spanned more than one heartbeat. Peak velocity through the aortic valve was assessed in $\mathrm{BH}$ and RT images and compared. Maximum peak velocity was used when $\mathrm{RT}$ images spanned more than one heartbeat.

\section{Results}

Example images from one patient are shown in Figure 1. There was good to excellent agreement between realtime imaging and breath-held imaging for all assessed parameters: the kappa statistic for agreement for LGE was 0.73 for reviewer $1(\mathrm{n}=204)$ and 0.64 for reviewer 2 $(n=202)$. Quantitative assessment of ejection fraction and peak aortic velocity revealed excellent concordance correlations of 0.89 and 0.91 respectively between breath-held and real-time acquisitions.

\section{Conclusions}

Prospective, real-time data acquisitions of left ventricular ejection fraction, late-Gadolinium enhancement, and quantitative velocity mapping reveal comparable, reliable, interpretable data compared to traditional breath-held CMR methodologies. These results augur well for improved CMR throughput and reliability in the future.

\section{Funding}

Grants from the National Institutes of Health (R01 HL102450) and Siemens Healthcare. 
Table 1 Imaging parameters for real time and breath held imaging

\begin{tabular}{|c|c|c|c|c|c|c|}
\hline & \multicolumn{6}{|c|}{ Typical imaging parameters } \\
\hline & \multicolumn{2}{|c|}{ Cine } & \multicolumn{2}{|c|}{ Velocity mapping } & \multicolumn{2}{|c|}{ Late gadolinium enhancement } \\
\hline & $\mathrm{BH}$ & RT & $\mathrm{BH}$ & RT & $\mathrm{BH}$ & RT \\
\hline Sequence Type & SSFP & SSFP & GRE & GRE-EPI & IR-GRE & IR-SSFP \\
\hline $\mathrm{TR} / \mathrm{TE} \mathrm{ms}$ & $2.9 / 1.2$ & $2.2 / 1.0$ & $5.3 / 2.1$ & 10.0/7.0 & $8.0 / 4.2$ & $2.8 / 1.1$ \\
\hline Matrix & $256 \times 256$ & $108 \times 160$ & $128 \times 192$ & $84 \times 128$ & $160 \times 192$ & $100 \times 192$ \\
\hline Slice Thickness (mm) & 8 & 10 & 6 & 10 & 8 & 8 \\
\hline Temporal Resolution (ms) & 40 & 60 & 53 & 40 & 200 & 180 \\
\hline Acceleration Rate & R2 & R4 & R2 & R3 & R1 & R2 \\
\hline Bandwidth (Hz/pixel) & 930 & 1488 & 401 & 2790 & 130 & 1184 \\
\hline Breath-hold Time (RR) & 11 & - & 16 & - & 12 & - \\
\hline
\end{tabular}
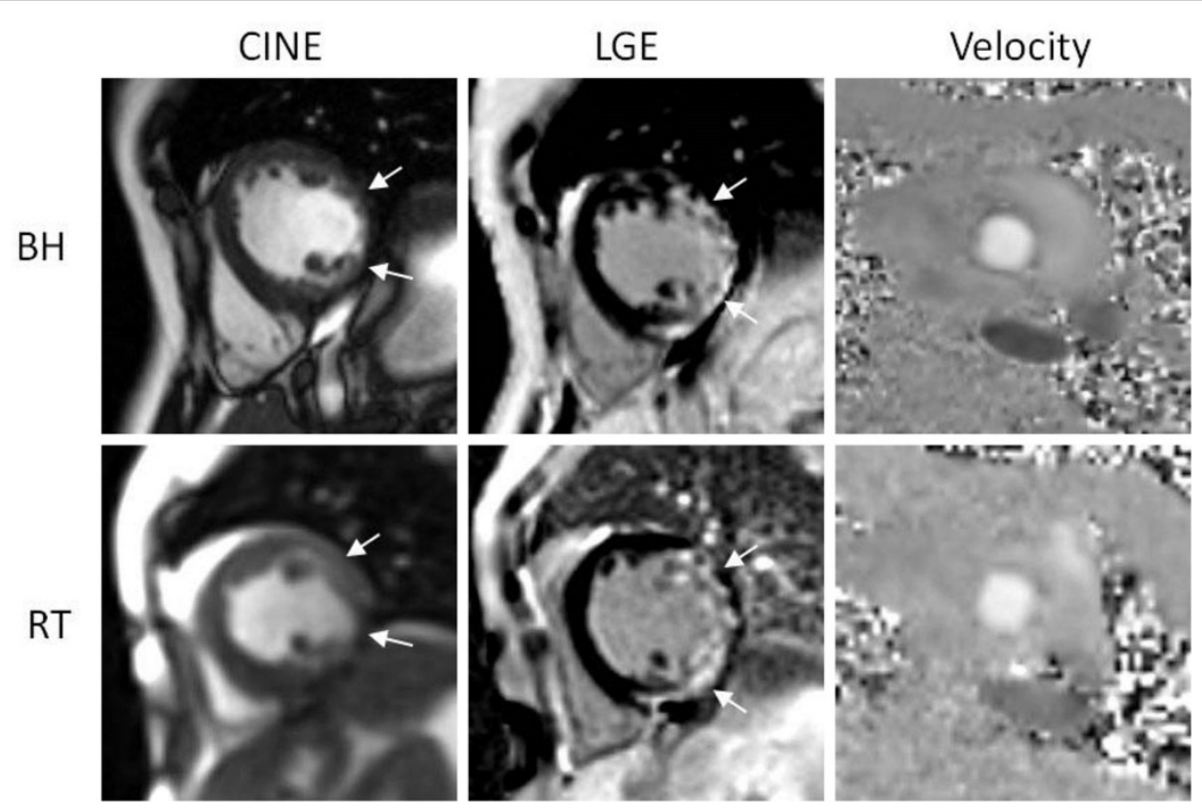

Figure 1 Representative example of cine, LGE and velocity breath-held and real-time imaging : arrows show areas of post-infract subendocardial scarring seen on real-time and breath-held images that had a corresponding lateral wall motion abnormality on both real-time and breath-held cine images.

\section{Author details}

'Department of Internal Medicine, Department of Cardiovascular Medicine, The Ohio State University Medical Center, Columbus, OH, USA. ${ }^{2}$ Siemens Healthcare, Columbus, OH, USA. ${ }^{3}$ Internal Medicine and Radiology, The Ohio State University, Columbus, OH, USA. ${ }^{4}$ Division of Biostatistics, College of Public Health, The Ohio State University, Columbus, OH, USA. ${ }^{5}$ Dorothy M. Davis Heart and Lung Research Institute, The Ohio State University, Columbus, OH, USA.

Published: 30 January 2013

doi:10.1186/1532-429X-15-S1-051

Cite this article as: Aneja et al:: Left ventricular function, aortic velocity, and late gadolinium enhancement assessed by real-time and single shot CMR is comparable to breath-held segmented imaging: a prospective study. Journal of Cardiovascular Magnetic Resonance 201315 (Suppl 1):051.

\section{Submit your next manuscript to BioMed Central} and take full advantage of:

- Convenient online submission

- Thorough peer review

- No space constraints or color figure charges

- Immediate publication on acceptance

- Inclusion in PubMed, CAS, Scopus and Google Scholar

- Research which is freely available for redistribution 